\title{
Clinical Use of Continuous Glucose Monitoring in Adults with Type 1 Diabetes
}

\author{
David Slattery, MD, MRCPI and Pratik Choudhary, MBBS, MD, FRCP
}

\begin{abstract}
With the emphasis on intensive management of type 1 diabetes, data from studies support frequent monitoring of glucose levels to improve glycemic control and reduce glucose variability, which can be related to an increase in macro and microvascular complications. However, few perform capillary blood glucose that frequently. There are currently two available alternatives that this review will discuss, continuous glucose monitoring (CGM) and flash glucose monitoring. CGM has become an important diagnostic and therapeutic option in optimizing diabetes management. CGM systems are now more accurate, smaller, and easier to use compared to original models. Randomized controlled trials (RCTs) have demonstrated that CGM can improve Hemoglobin A1c (HbA1C) and reduce glucose variability in both continuous subcutaneous insulin infusion and multiple daily injection users. When used in an automated "insulin-suspend" system, reduced frequency of hypoglycemia and shorter time spent in hypoglycemic range have been demonstrated. Despite the potential benefits CGM has to offer in clinical practice, concerns exist on the accuracy of these devices and patient compliance with therapy, which may prevent the true clinical benefit of CGM being achieved, as observed in RCTs. Flash glucose monitoring systems FreeStyle ${ }^{\circledR}$ Libre $^{\mathrm{TM}}$ (Abbott Diabetes Care, Alameda, CA) are as accurate as many CGM systems available and have the added benefit of being factory calibrated. Studies have shown that flash glucose monitoring systems are very well tolerated by patients and effectively reduce glucose variability, increasing time in range.
\end{abstract}

Keywords: Type 1 diabetes, Continuous glucose monitoring, Flash glucose monitoring, Hypoglycemia, Glycemic control.

\section{Introduction}

$\mathbf{I}^{\mathrm{T}}$ NTENSIVE MANAGEMENT OF type 1 diabetes mellitus (T1DM), with multiple daily injections (MDI) or continuous subcutaneous insulin infusion (CSII), using principles of functional insulin therapy as per DAFNE or ITTP has been shown to improve outcome by reducing the risk of developing micro and macrovascular complications (DCCT group). ${ }^{1}$ Technologies have developed at an accelerated rate over the past three decades to aid in this intensive approach to managing type 1 diabetes (T1D), with the end goals of improving glycemic control and reducing frequency of problematic hypoglycemia. CSII and continuous glucose monitoring (CGM) therapy have been at the forefront of this technological advancement. The concept of interstitial CGM has existed since the 1990s with the first system being released by Medtronic in 1999 (CGMS ${ }^{\circledR}$ Gold; Medtronic, Inc., Northridge, $\mathrm{CA})^{2}$

CGM can be divided into two categories. Blinded retrospective CGM done intermittently used to collect data on glucose excursion, look for patterns, and facilitate changes in therapy or real-time CGM, displaying current glucose value in addition to direction and rate of change (ROC), equipped with alerts and alarms to facilitate prompt real-time actions in response to impending hyper/hypoglycemia. These measure interstitial glucose, with a lag of 4-10 min and usually require between two to four calibration tests (capillary blood glucose [CBG] values) to ensure accuracy. ${ }^{3}$

Flash glucose monitoring systems, FreeStyle ${ }^{\circledR}$ Libre $^{\mathrm{TM}}$ (Abbott Diabetes Care, Alameda, CA) have recently been

Weston Education Centre, Kings College London, London, United Kingdom. 
introduced and are designed to replace CBG. Like CGM, flash glucose monitoring provides real-time interstitial glucose levels and trends of glucose levels, however, these systems do not alarm. They have the advantage of being factory calibrated so the user does not have to do any $\mathrm{CBG}$, although the label does suggest confirmatory CBG in case of hypoglycemia or if rapid fluctuation. These systems can also send glucose values to an app on an Android ${ }^{\circledR}$ device (Google, Mountain View, CA), removing the need for a separate reader.

The potential advantages that these glucose monitoring systems may have, appear obvious to see. As CGM usage is not routinely reimbursed by the majority of international healthcare systems, strict clinical criteria exist for determining who would benefit from having CGM as part of their treatment regimen. We will discuss the clinical indications and the evidence that supports CGM use in T1DM management, along with limitations, which have been highlighted in the literature.

\section{CGM for Glucose Monitoring}

Monitoring of glucose levels is essential for achieving target glycemic control and avoiding hypoglycemia, especially in patients with T1DM on MDI or CSII therapy. The positive relationship between frequency of CBG and metabolic control is established. ${ }^{4}$ The T1D exchange registry showed that those in the excellent control group ( $\mathrm{HbA} 1 \mathrm{C}<6.5 \%)$ more frequently performed CBG compared to the fair/poor control group ( $\geq 8.5 \%)$ ( $72 \%$ vs. $36 \%$ reporting $\mathrm{CBG}$ frequency $\geq 5$ times/day) respectively, including more frequent CBG measurements before bolusing ( $56 \%$ vs. $32 \%)^{5}$

Key benefits of CGM monitoring:

\section{(1) Frequency of testing}

Although these data support frequent measurement, the clinical reality is that many people do not do so, for a variety of reasons. Even though the technology for CBG has improved and the actual measurement takes less than a few seconds, there is a complex procedure, let alone the pain, social embarrassment, and inconvenience caused. A key benefit from CGM is just the frequency at which people look at their data.

(2) Trends

The second benefit is knowing the direction of change, so that the patient can take different action for a glucose value that is rising as opposed to falling. ${ }^{6}$

(3) Alarms

Alarms can help alert patients to times when they are going out of range or at levels that they are uncomfortable with. This is especially relevant for hypoglycemia. These variations can occur due to a variety of reasons such as miscounting of carbohydrates, effect of activity, and effect of stress to name a few.

(4) Therapy optimization

CGM provides a complete dataset for clinicians and patients to look for patterns and trends allowing for identification of postprandial peaks, nocturnal hypoglycemia, or the dawn phenomenon. ${ }^{7}$

(5) Diagnosis

CGM is increasingly used to identify glucose fluctuations or hypoglycemia in areas such as cystic fibrosis-related diabetes, insulinomas, and post-bariatric surgery hypoglycemia. ${ }^{8-10}$

\section{CGM for Guiding Diabetes Management}

CGM systems can be used blinded or open. When we are using CGM short-term to identify trends, such as postprandial glucose peaks, hypoglycemia, and dawn phenomenon, it is often cleaner to use blinded CGM where data are not displayed to the patient and therefore cannot be used to influence the readings. These data can be reviewed retrospectively by both the patient and physician, acting as a diagnostic tool.

Open systems display glucose levels, trends, and alerts on the receiver in real-time, along with having alarms and allow the patient to intervene and steer their diabetes management. New technological developments now enable some CGM sensors to transmit signals to the "cloud," with such systems as the Guardian Connect system or Dexcom G4 and G5 systems (Dexcom, Inc., San Diego). Night scout (CGM in the cloud) is an open-source DIY project developed by users that allows real-time access to CGM data via a personal website, or to smart watches, which has over 17,000 users. ${ }^{11}$

Several randomized controlled trials (RCTs) have evaluated the potential benefit of CGM as guidance for treatment. The Guard Control study in 2006 evaluated 162 children with suboptimal glycemic control (initial HbA1c 9.6\% $\pm 1.2 \%$ ) over a period of 3 months. Even though this early system did not have alarms, and accuracy was much lower than current systems, there was a significant improvement in HbA1c of $0.6 \%$ in those with occasional use and a much greater reduction in patients with the highest rate of use $(1.0 \% \pm 1.1 \%)$. This was achieved without an increase in the number of hypoglycemic episodes. Twenty-six percent achieved a reduction of $\geq 2 \%$. Occasional use of the CGM system led to a mean improvement in HbAlc of 0.6. ${ }^{12}$

The Juvenile Diabetes Research Foundation (JDRF) landmark study randomized 322 adults, adolescents, and children with T1DM into three age groups: children aged 814 years, young adults aged 15-24 years, and adults of 25 years and older. CGM use for 26 weeks significantly reduced $\mathrm{HbAlc}$ by $0.5 \%$ in adult patients, without any increase in hypoglycemia. However, the younger participants $(<25$ years) did not show significant improvement, which was likely related to $<50 \%$ adherence in these groups. Of note, $90 \%$ of patients in this study were using CSII as well. ${ }^{13}$

Battelino et al. compared CGM versus CBG in 120 wellcontrolled patients with T1DM (baseline HbA1c 6.9\%) for 26 weeks. The HbA1c declined in the CGM group to $6.69 \%$ $(P=0.008)$, while it remained unchanged in the control group, at $6.95 \% .^{14}$

The technology has been evolving and later studies evaluated the effect of CGM therapy on glycemic control exclusively in subjects on MDI treatment. The GOLD study was a randomized crossover study using Dexcom G4 Platinum system in patients with T1DM with an $\mathrm{HbA1C}$ of $\geq 7.5 \%$ on MDI. HBA1C was significantly lower with CGM use compared to CBG (7.92 [0.8] vs. 8.35 [0.9]). Adherence to CGM treatment was excellent in this study (the overall mean time of CGM use was $87.8 \%$ ). ${ }^{15}$

The DIAMOND study was a two-arm randomized controlled trial of CGM (Dexcom G4 Platinum vs. CBG) over 24 weeks in a similar population of T1D treated with MDI with $\mathrm{HbA} 1 \mathrm{C}$ levels between $7.5 \%$ and $9.9 \%$. Mean $\mathrm{HbA1C}$ reduction from baseline was $1.1 \%$ at 12 weeks and $1.0 \%$ at 24 weeks in the CGM group and $0.5 \%$ and $0.4 \%$, respectively, in 
the control group (repeated-measures model $P<0.001$ ). In the CGM group, full compliance was $93 \%$ in month $6 .{ }^{16}$

What the above studies demonstrate is that continuous use and high levels of compliance are a necessity, if maximum glucose-lowering effect with CGM is to be achieved. The DIAMOND and GOLD studies also show the benefit of CGM in people using conventional MDI treatment, which the majority of patients with T1DM use.

The recently published REPLACE-BG study demonstrated that CGM data could be used to make safe treatment decisions, showing no difference at all in those using CGM alone for decision making versus those using a confirmatory CBG reading. Mean time in $70-180 \mathrm{mg} / \mathrm{dL}$ was $63 \% \pm 13 \%$ at both baseline and 26 weeks in the CGM-only group and $65 \% \pm 13 \%$ and $65 \% \pm 11 \%$ in the CGM+CBG group. No severe hypoglycemic events occurred in the CGM-only group, and one occurred in the CGM+BGM group. ${ }^{17}$

The results of a large randomized clinical trial known as IMPACT were published in 2016 in The Lancet by Bolinder et al. This study demonstrated that patients who were randomized to flash CGM for glucose monitoring accessed glucose values on average 15.1 times per day versus control subjects who monitored via CBG on average 5.6 times per day. Furthermore, reductions in hypoglycemic events at each of three distinct thresholds were observed: 70,55 , and $45 \mathrm{mg} / \mathrm{dL}$. These reductions were statistically significant. Improvements in time in range $(70-180 \mathrm{mg} / \mathrm{dL})$ and glucose variability were also seen. ${ }^{18}$

Other recent studies have highlighted the potential for HbA1c improvement with flash CGM technology. In a study by Dover et al., mean HbA1c decreased from $8.0 \% \pm 0.14 \%$ to $7.5 \% \pm 0.14 \%$ after a 16 week period of flash CGM use in T1DM. ${ }^{19}$ Ish-Shalom et al. also noted an HbA1c decrease of $1.33 \% \pm 0.29 \%$ after 8 weeks of flash CGM use in a mixed population of people with difficult-to-control T1DM and T2DM. ${ }^{20}$ McKnight and Gibb reported a significant change in $\mathrm{HbA} 1 \mathrm{c}$ among flash CGM users versus nonusers with T1DM in Scotland $\left(-0.2 \%\right.$ versus $+0.1 \%$, respectively) ${ }^{21}$ Although these are small non-controlled observational studies, they add to the emerging body of evidence supporting beneficial clinical outcomes with the use of flash CGM in people with T1DM.

\section{CGM for Preventing Hypoglycemia}

Impaired awareness of hypoglycemia (IAH) and severe hypoglycemia $(\mathrm{SH})$ are significant contributors to morbidity associated with long-term insulin therapy. ${ }^{22,23}$ In many countries, including the United Kingdom, CGM is approved for use in cases of problematic hypoglycemia (NICE). This is supported by RCTs carried out in patients with T1DM in which the primary end points were time spent in low glucose range or prevention of hypoglycemic episodes.

In the JDRF $<7$ study, patients with good control were included (HbA1c <7.0\%). In the intervention group (CGM for 6 months), time spent in the hypoglycemic range of $<70 \mathrm{mg} / \mathrm{dL}$ was reduced by $41 \%$, compared to the control group (CBG) who had no change. However, there was no significant difference in the number of hypoglycemic episodes or $\mathrm{SH}$ between the groups along with $\mathrm{HbA1C}$ remaining static. In this study, CGM use dropped from $78 \%$ of the time in the first 4 weeks to $67 \%$ in the final 4 weeks, which may explain why $\mathrm{HbA} 1 \mathrm{C}$ and frequency of hypoglycemia and $\mathrm{SH}$ remain unchanged. ${ }^{24}$
The IN CONTROL study by Van Beers, evaluated the ability of CGM to reduce SH in a high risk group with IAH (Gold score $>4$ ), in a randomized crossover study. There was a reduction in time spent in hypoglycemia $(6.8 \%$ vs. $11.4 \%)$ with significantly fewer severe hypoglycemia events during the CGM arm than CBG (14 vs. 34 events, $P=0.033$ ). However, rates went back to baseline on those who used CGM first, suggesting there was no learning effect, and the effect only lasted while CGM was being used. ${ }^{25}$

In the Battelino study mentioned above, the time per day spent in hypoglycemia was significantly shorter in the CGM group than in the control group $(0.48 \pm 0.57$ and $0.97 \pm 1.55 \mathrm{~h} /$ day, respectively, $P=0.03) .{ }^{14}$ In the GOLD study, hypoglycemia was less in the CGM group compared to the conventional control group $(2.79 \%$ vs. $4.79 \%$, respectively). There were fewer (5 vs. 1) SH events during CGM therapy. ${ }^{15}$ The DIAMOND study also demonstrated a favorable effect on hypoglycemia with CGM use, with median duration of hypoglycemia at less than $43 \mathrm{~min} /$ day in the CGM group versus $80 \mathrm{~min} /$ day in the control group $(P=0.002)$. However, there was no difference in SH between the two groups ( 2 vs. 2 ). ${ }^{16}$

One might argue that CGM is equally as effective at lowering time spent in hypoglycemic range as it is at lowering HbA1C. A key factor in earlier studies has been compliance, but with greater compliance seen with newer devices, we are getting more consistent benefits. There are, however, few data on high risk groups such as those with $\mathrm{IAH}$, and none in this group with flash monitoring.

\section{CGM with Sensor-Augmented Pump Therapy}

When used with CSII, CGM can be used independently to guide therapy or integrated with an insulin pump, sensoraugmented pump (SAP) therapy. Studies that looked at the potential benefit of SAP therapy without LGS on glycemic control included RealTrend, Eurythmics, STAR 3, and SWITCH. The RealTrend study looked at adults and children with T1DM (HbA1C $\geq 8 \%$ ) on MDI. They were randomized to insulin pump alone, or SAP with instructions to wear CGM sensors at least $70 \%$ of the time. HbA1C improved significantly in both groups (CGM group $-0.81 \% \pm 1.09 \%, P<0.001$; CSII group $-0.57 \% \pm 0.94 \%, P<0.001$ ), with no significant difference between groups. However, when sub-analyzing only the patients who were fully compliant with sensor use, a significant improvement in $\mathrm{HbA} 1 \mathrm{C}$ was observed when compared to the CSII alone group $(-0.96 \% \pm 0.93 \%, P<0.001$; CSII group $-0.55 \% \pm 0.93 \%, P<0.001) .{ }^{26}$ The Eurythmics trial randomized 83 patients with T1DM being treated with MDI, aged $18-65$ years with $\mathrm{HbA1c} \geq 8.2 \%$ to 26 weeks of treatment with either a SAP $(n=44)$ or continued MDI therapy $(n=39)$. Mean HbA1C remained unchanged in the control arm ( $8.59 \%$ to $8.46 \%$ ) but was significantly reduced in the SAP group $(8.46 \%$ to $7.23 \%){ }^{27}$

STAR-3 examined the effects of crossing over from optimized MDI therapy to SAP therapy for 6 months, and the effects of 18 months' sustained use of SAP. The primary outcome was change in $\mathrm{HbA} 1 \mathrm{C}$ in the crossover group. HbA1C values were initially lower in the continuing-SAP group than in the crossover group ( $7.4 \%$ vs. $8.0 \%, P<0.001)$. HbA1C values remained reduced in the SAP group. After 3 months on the SAP system, HbA1C decreased to $7.6 \%$ in the crossover group 
$(P<0.001)$; this was a significant and sustained decrease among both adults and children $(P<0.05) .^{28}$

The SWITCH trial investigated children and adults with T1DM with HbA1C between $7.5 \%$ and $9.5 \%$ using CSII alone. They were randomized to CGM sensor-on or sensoroff arms for 6 months, then crossed over. The mean difference in $\mathrm{HbA1c}$ was $-0.43 \%$ in favor of sensor-on arm. Stopping CGM system use resulted in $\mathrm{HbA1c}$ reverting to baseline levels. Median time spent in hypoglycemia (<70 mg/ dL) was far less with sensor switched on versus off (19 min vs. $31 \mathrm{~min}$ ), with more time spent in euglycemic range $(774 \pm 232$ vs. $669 \pm 208)$. However, patients went back to baseline in the CGM first arm, suggesting that the benefits only lasted as long as the CGM was used. ${ }^{29}$

These studies show that SAP therapy can offer superior glycemic control when compared to pump therapy alone or MDI, with good sensor compliance as highlighted by the RealTrend study. However, not all studies demonstrated a reduction in hypoglycemia. In fact, in the eurythmics trial, there were four episodes of SH in the SAP group compared to one in the MDI group.

\section{CGM with Low Glucose Suspend Sensor Augmented Pump Therapy}

SAP therapy may also have a low glucose suspend (LGS) feature which automatically stops insulin delivery in response to hypoglycemia. The MiniMed 640G SAP system (Medtronic, Inc.) can automatically suspend insulin delivery in advance of predicted hypoglycemia and restart it upon recovery. Several studies have demonstrated clear, clinical benefits of SAP therapy in diabetes management and can be subdivided into two categories of having LGS or not.

The ASPIRE home study was a large multicenter study evaluating LGS against SAP. There was a $27.5 \%$ reduction in mean AUC for nocturnal hypoglycemic events in the LGS, showing a clear benefit in preventing nocturnal hypoglycemia. The mean AUC for combined daytime and nighttime hypoglycemic events was $31.4 \%$ lower in the LGS group than in the control group. Four SH were reported in the control group and none in the LGS group. ${ }^{30}$

Danne et al. evaluated the same system in children, with T1DM. The number of hypoglycemic excursions (average/day) was reduced with SAP+LGS, compared to SAP $(<70 \mathrm{mg} / \mathrm{L}$, $1.27 \pm 0.75$ vs. $0.95 \pm 0.49, P=0.010 ; \leq 40 \mathrm{mg} / \mathrm{dL}, 0.28 \pm 0.18$ vs. $0.13 \pm 0.14, P=0.005)$ as was the time spent in hypoglycemia (average min/day, $101 \pm 68$ vs. $58 \pm 33, P=0.002$ ) without significant difference in the mean glucose level $(145 \pm 23 \text { vs. } 148 \pm 19 \mathrm{mg} / \mathrm{dL})^{31}$

Choudhary et al. evaluated the LGS feature of the Paradigm Veo insulin pump (Medtronic, Inc.) for 3 weeks in 31 adults with T1D. There were 166 episodes of LGS: $66 \%$ of daytime LGS episodes were terminated within $10 \mathrm{~min}$, and 20 episodes lasted the maximum $2 \mathrm{~h}$. LGS use was associated with reduced nocturnal duration $\leq 2.2 \mathrm{mmol} / \mathrm{L}$ in those in the highest quartile of nocturnal hypoglycemia at baseline (median 46.2 vs. $1.8 \mathrm{~min} /$ day, $P=0.02$ [LGS-OFF vs. LGS-ON]). ${ }^{32}$

Ly et al., performed a large RCT in children and young adults with IAH, comparing CSII with SAP+LGS. The event rates decreased from 28 to 16 in the pump-only group versus 175 to 35 in the LGS group. However, despite randomization, a significant baseline difference in the prevalence of hypoglycemia existed between the control and LGS group (20.7 vs. 129.6 events per 100 patient months). Also, results could not be deemed reliable due to the use of event rates. ${ }^{33}$

The next generation of systems suspends insulin delivery in response to predicted hypoglycemia (Medtronic 640G; Medtronic, Inc.). This predictive LGS system was evaluated in 40 patients with T1DM. There were 2322 suspend before low events ( 2.1 per subject-day). Sensor glucose values following $1930(83.1 \%)$ of the predictive suspensions did not reach the preset low limit. Nadir sensor glucose values of $\leq 50$ and $\leq 60 \mathrm{mg} / \mathrm{dL}$ were seen in $207(8.9 \%)$ and $356(15.3 \%)$ of the predictive suspensions, respectively. ${ }^{34}$ Importantly, there was no restoration of awareness in these patients, even though SH was reduced. This may be because the LGS system, only activated once hypoglycemia had occurred and did not actually prevent biochemical hypoglycemia, and so awareness was not restored. Analysis of data uploaded by over 5000 users into the Medtronic Carelink database from across Europe found that less than a quarter of the "predicted low" events reached the low glucose threshold. Duration of night time hypoglycemia was significantly lower in those patients with suspend on low feature activated $(0.4 \pm 0.8$ vs. $0.2 \pm 0.5 \mathrm{~h} ; P<0.001)$; and lower avain when using the suspend before low feature $(0.4 \pm 1.0$ vs. $0.1 \pm 0.1 \mathrm{~h} / \mathrm{night}$; $P<0.001){ }^{35}$

\section{CGM to Improve Quality of Life}

While the primary end points in many of the studies discussed so far on CGM focus on improvements in glycemic control and prevention of hypoglycemia, measuring the impact a therapy has on quality of life is also key.

The GOLD study showed a significant improvement in QoL in CGM-treated patients when compared to controls. Patient well-being assessed using the WHO-5 questionnaire improved with CGM therapy (66.1 vs. 62.7, $P=0.02)$. Treatment satisfaction with CGM measured using the Diabetes Treatment Satisfaction Questionnaire (DTSQ) improved (30.21 vs. $26.62, P<0.001)$ as it did with the change version (13.20 vs. 5.97, $P<0.001)$. The Hypoglycemia Confidence Questionnaire (HCQ) scale showed less hypoglycemia fear with CGM use $(3.40$ vs. $3.27, P<0.001) .{ }^{15}$

In the DIAMOND study, the CGM group had a mean score of 4.2 (0.4) with the CGM satisfactory survey, with mean scores of $4.2(0.5)$ on the benefits subscale and $4.3(0.5)$ on the lack of hassles subscale. ${ }^{16}$

The JDF CGM study also found a slight $(P<0.05)$ improvement in QoL with CGM for participants $\geq 18$ years old. There were improvements in Hypoglycemia Fear Survey and Social Functioning Health Survey (SF-12). There were no differences in scores for youth or their parents for any measures after 26 weeks, although as mentioned, CGM use was low in this group. CGM satisfactory scores at 26 weeks were higher than neutral (3.0) for adults, youth, and parents. ${ }^{36}$

\section{Limitations of CGM}

\section{Adherence}

A common finding in CGM studies is that adherence to therapy yields better glycemic control, this is evident in the 
JDRF and RealTrend studies and Pickup metanalysis. ${ }^{24,26,37}$ Aspire, IMPACT, DIAMOND, and Gold have all shown that $>80 \%$ compliance was required to receive optimum benefit with all major available systems. What is also evident is that $\mathrm{SH}$ is not prevented with intermittent compliance. The HYPOCOMPASS study carried out in a cohort of patients with IAH, showed that overall sensor usage was around $50 \%$ of the time, with $17 \%$ of patients using the sensor $>80 \%$ of the time. CGM was not associated with an improvement in IAH or SH in this study. ${ }^{38}$

Wong et al., assessed CGM use in the T1D exchange registry. Of the 1662 participants reporting CGM use at enrollment into the registry, $675(41 \%)$ reported discontinuing CGM after 1 year. The main reason for stopping CGM was discomfort when wearing the CGM (42\%), followed by problems with CGM insertion (33\%), problems with adhesion to skin (30\%), poor performance (28\%), alarms $(27 \%)$, accuracy $(25 \%)$ interference with sports and activities (18\%), and skin reactions from the CGM sensor $(18 \%))^{39}$ However, with the advancement of CGM technology in recent years, newer generation devices have resulted in improved compliance, better tolerability, and patient satisfaction being much higher.

Flash glucose monitoring has been very tolerated in clinical studies to date. In the study by Bailey et al., 98.6\% of sensor insertions had a pain rating $\leq 2$ (favorable response), $99.3 \%$ offered the same rating for acceptable amount of bleeding on sensor insertion, $0.5 \%$ reported itching, and $4 \%$ reported mild erythema. Taking these into account, along with factory calibration and no alarm system, flash glucose monitoring could offer much higher rates of compliance and is an attractive option. ${ }^{40}$

\section{Accuracy}

The performance and accuracy of CGM is a concern and about $25 \%$ of CGM users report accuracy as a major reason for discontinuing treatment. Accuracy is measured using the mean absolute relative differences (MARDs) between CGM readings and $\mathrm{BG}$ readings. Early $\mathrm{CGM}$ devices had quite a high error rate with MARDs of around 20\% reported, however, with advances in sensor technology, these rates are now as low as between $9 \%$ and $14 \%$. The MARD of the flash glucose monitoring system is $11.4 \%$ and that of the latest Dexcom G5 system is just under $10 \%$. Contributing factors to this inaccuracy that is sometimes observed include the physiological lag time, which exists between interstitial and blood glucose. This lag time is between 4 and 10 min and can be longer when glucose concentrations are changing rapidly. ${ }^{3}$ Also, when the descent of glucose levels is rapid on approaching hypoglycemic levels, sensor glucose can be higher than blood glucose, resulting in false reassurance.

\section{Optimizing value from CGM}

Since technology has improved, so has accuracy. Patient acceptance has risen as evidenced by the improved acceptance of newer systems such as Freestyle Libre and Dexcom in their latest studies. We are also getting better at showing

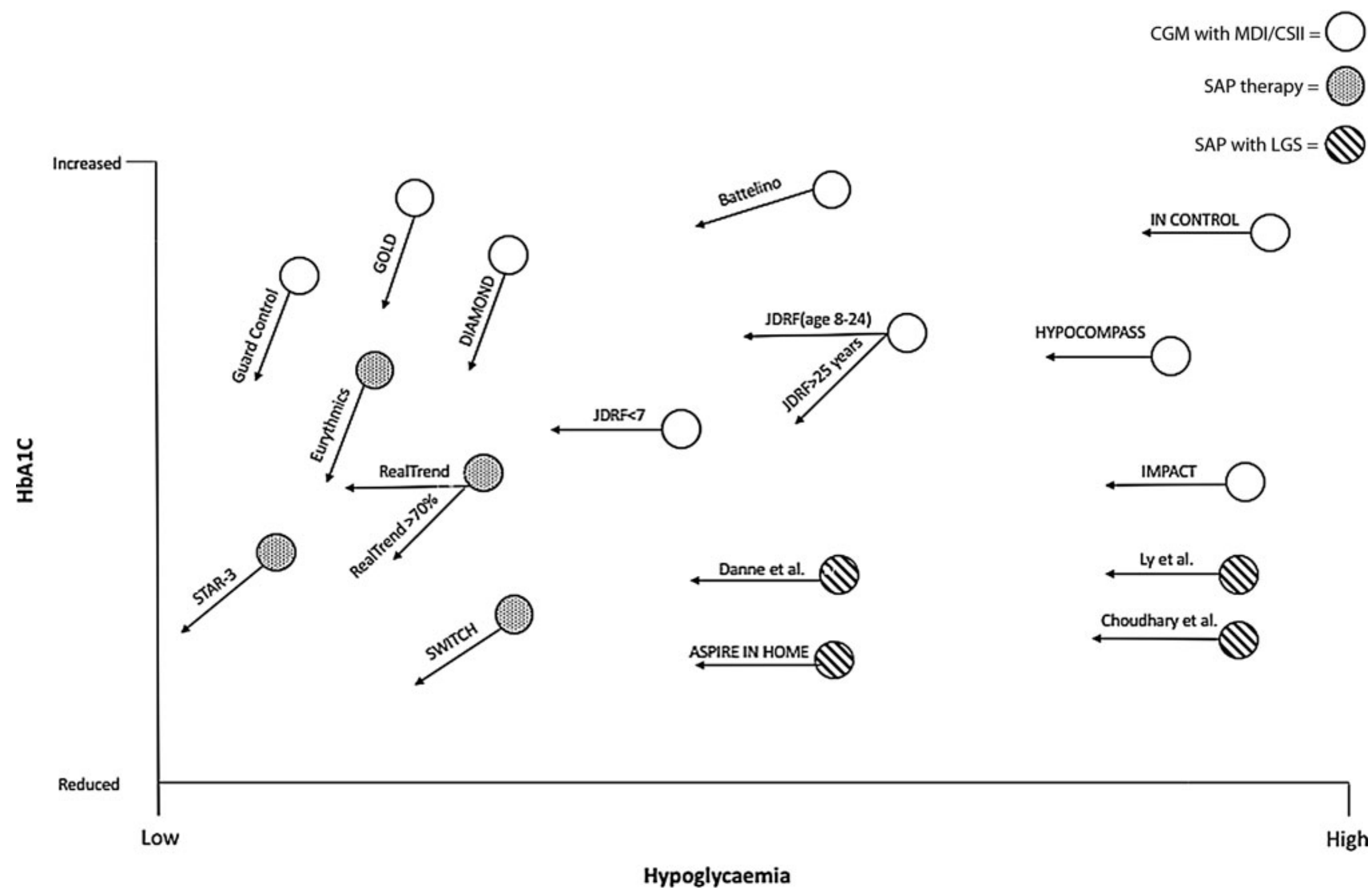

FIG. 1. Graphical representation of CGM studies. CGM, continuous glucose monitoring; CSII, continuous subcutaneous insulin infusion; MDI, multiple daily injections; SAP, sensor-augmented pump. 
patients how to use the systems, and how to use the increased information such as trend information in a valuable way.

A study by Pettus and Edelman investigated how CGM was utilized by people with T1DM and whether any differences were seen between MDI versus CSII users, with particular focus on ROC arrows to guide insulin therapy. This study showed that CGM users regardless of type of therapy, relied heavily on ROC information. Most CGM users reported an increase in the number of daily injections/ boluses, with MDI users taking fewer boluses when compared to CSII. The majority of CGM users in this study also reported using ROC arrows frequently to guide changes in their insulin dosage, with these changes more aggressive than the recommended $10 \%-20 \%$ adjustments commonly advised. ${ }^{6}$

\section{Summary}

The evidence from RCTs is summarized in Figure 1 and has shown us that CGM is very effective and a useful tool in lowering HbA1C, when used with either CSII or MDI. When used in a SAP system the benefits can again be seen, however, perhaps the greatest advantage from using CGM is with LGS and preventing hypoglycemia. While CGM offers a really valuable therapeutic option, it does not work for all patients. High levels of compliance and interaction with devices are necessary with current CGM systems, to see maximum potential benefit, as demonstrated by some studies. One might argue that people who participated in these CGM studies were highly motivated and in reality, compliance would not be as good, as highlighted in the T1D exchange registry. Flash glucose monitoring systems are an attractive option. They are extremely accurate, well tolerated, and easy to use.

The cost of CGM also poses a barrier to usage. Despite the obvious advantages these devices may have, they are not widely available for use by people with diabetes mellitus. Cost restrictions by most national health systems mean that CGM is funded on an individual case by case basis and only if a patient meets strict criteria for usage such as not being able to achieve target glycemic control with conventional therapy or problematic hypoglycemia. While patients do have the option to self-fund these sensors, they remain quite expensive.

\section{References}

1. The Diabetes Control and Complications Trial Research Group, Nathan DM, Genuth S, et al.: The effect of intensive treatment of diabetes on the development and progression of long-term complications in insulin-dependent diabetes mellitus. N Engl J Med 1993;329:977-986.

2. Mastrototaro J: The miniMed continuous glucose monitoring system (CGMS). J Pediatr Endocrinol Metab 1999; 12:751-758.

3. Boyne MS, Silver DM, Kaplan J, et al.: Timing of changes in interstitial and venous blood glucose measured with a continuous subcutaneous glucose sensor. Diabetes 2003;52: 2790-2794.

4. Schütt M, Kern W, Krause U, et al.: Is the frequency of self-monitoring of blood glucose related to long-term metabolic control? Multicenter analysis including 24,500 patients from 191 centers in Germany and Austria. Exp Clin Endocrinol Diabetes 2006;114:384-388.

5. Simmons JH, Chen V, Miller KM, et al.: Differences in the management of type 1 diabetes among adults under excellent control compared with those under poor control in the T1D Exchange Clinic Registry. Diabetes Care 2013;36: 3573-3577.

6. Pettus J, Edelman SV: Use of glucose rate of change arrows to adjust insulin therapy among individuals with type 1 diabetes who use continuous glucose monitoring. Diabetes Technol Ther 2016;18 Suppl 2:S234-S242.

7. Kaufman FR, Gibson LC, Halvorson M, et al.: A pilot study of the continuous glucose monitoring system: clinical decisions and glycemic control after its use in pediatric type 1 diabetic subjects. Diabetes Care 2001;24:2030-2034.

8. Schiaffini R, Brufani C, Russo B, et al.: Abnormal glucose tolerance in children with cystic fibrosis: the predictive role of continuous glucose monitoring system. Eur J Endocrinol 2010;162:705-710.

9. Munir A, Choudhary P, Harrison B: Continuous glucose monitoring in patients with insulinoma. Clin Endocrinol (Oxf) 2008;68:912-918.

10. Kefurt R, Langer FB, Schindler K, et al.: Hypoglycemia after Roux-en-Y gastric bypass: detection rates of continuous glucose monitoring (CGM) versus mixed meal test. Surg Obes Relat Dis 2015;11:564-569.

11. www.nightscout.info (accessed January 25, 2017).

12. Deiss D, Bolinder J, Riveline JP, et al.: Improved glycemic control in poorly controlled patients with type 1 diabetes using real-time continuous glucose monitoring. Diabetes Care 2006;29:2730-2732.

13. Juvenile Diabetes Research Foundation Continuous Glucose Monitoring Study Group, Tamborlane WV, Beck RW, et al.: Continuous glucose monitoring and intensive treatment of type 1 diabetes. N Engl J Med 2008;359:14641476.

14. Battelino T, Phillip M, Bratina N, et al.: Effect of continuous glucose monitoring on hypoglycemia in type 1 diabetes. Diabetes Care 2011;34:795-800.

15. Lind M, Polonsky W, Hirsch IB, et al.: Continuous glucose monitoring vs conventional therapy for glycemic control in adults with type 1 diabetes treated with multiple daily insulin injections: the GOLD randomized clinical trial. JAMA 2017;317:379-387.

16. Beck RW, Riddlesworth T, Ruedy K, et al.: effect of continuous glucose monitoring on glycemic control in adults with type 1 diabetes using insulin injections: the DIAMOND randomized clinical trial. JAMA 2017;317:371-378.

17. Aleppo G, Ruedy KJ, Riddlesworth TD, et al.: REPLACEBG: a randomized trial comparing continuous glucose monitoring with and without routine blood glucose monitoring in adults with well-controlled type 1. Diabetes Care 2017;40:538-545.

18. Bolinder J, Weitgasser R, Antuna R, et al.: Randomised controlled study to evaluate the impact of novel glucosesensing technology on hypoglycemia in type 1 diabetes. Lancet 2016. DOI: 10.1016/S0140-6736(16)31535-5.

19. Dover A, Stimson R, Zammitt N, Gibb F: Flash glucose monitoring improves outcomes in a type 1 diabetes clinic. $\mathbf{J}$ Diabetes Sci Technol 2017;11:442-443.

20. Ish-Shalom M, Wainstein J, Raz I, Mosenzon O: Improvement in glucose control in difficult- to-control patients with diabetes using a novel Flash glucose monitoring device. J Diabetes Sci Technol 2016;10:1412-1413. 
21. McKnight J, Gibb F: Flash glucose monitoring is associated with improved glycaemic control but use is largely limited to more affluent people in a UK diabetes centre. Diabet Med 2017. DOI: 10.1111/dme.13315.

22. Langan SJ, Deary IJ, Hepburn DA, Frier B: Cumulative cognitive impairment following recurrent severe hypoglycaemia in adult patients with insulin-treated diabetes mellitus. Diabetologia 1991;34:337-344.

23. Bree A, Puente E, Daphna-Iken D, Fisher S: Diabetes increases brain damage caused by severe hypoglycemia. Am J Physiol Endocrinol Metab 2009;297:E194-E201.

24. Juvenile Diabetes Research Foundation Continuous Glucose Monitoring Study Group, Beck RW, Hirsch IB, et al.: Glucose monitoring in well-controlled type 1 diabetes. Diabetes Care 2009;32:1378-1383.

25. van Beers CAJ, DeVries JH, Kleijer SJ: Continuous glucose monitoring for patients with type 1 diabetes and impaired awareness of hypoglycaemia (IN CONTROL): a randomised, open-label, crossover trial. Lancet Diabetes Endocrinol 2016;4:893-902.

26. Raccah D, Sulmont V, Reznik Y, et al.: Incremental value of continuous glucose monitoring when starting pump therapy in patients with poorly controlled type 1 diabetes: the RealTrend study. Diabetes Care 2009;32:2245-2250.

27. Hermanides J, Nørgaard K, Bruttomesso D, et al.: Sensoraugmented pump therapy lowers $\mathrm{HbA}(1 \mathrm{c})$ in suboptimally controlled type 1 diabetes; a randomized controlled trial. Diabet Med 2011;28:1158-1167.

28. Bergenstal RM, Tamborlane WV, Ahmann A, et al.: Effectiveness of sensor-augmented insulin-pump therapy in type 1 diabetes. N Engl J Med 2010;363:311-320.

29. Battelino T, Conget I, Olsen B, et al.: The use and efficacy of continuous glucose monitoring in type 1 diabetes treated with insulin pump therapy: a randomised controlled trial. Diabetologia 2012;55:3155-3162.

30. Richard M. Bergenstal, David C, et al.: Threshold-based insulin-pump interruption for reduction of hypoglycemia. $\mathrm{N}$ Engl J Med 2013;369:224-232.

31. Danne T, Kordonouri O, Holder M, et al.: Prevention of hypoglycemia by using low glucose suspend function in sensor-augmented pump therapy. Diabetes Technol Ther 2011;13:1129-1134.

32. Choudhary P, Shin J, Wang Y, et al.: Insulin pump therapy with automated insulin suspension in response to hypoglycemia: reduction in nocturnal hypoglycemia in those at greatest risk. Diabetes Care 2011;34:2023-2025.

33. Ly TT, Nicholas JA, Retterath A, et al.: Effect of sensoraugmented insulin pump therapy and automated insulin suspension vs standard insulin pump therapy on hypoglycemia in patients with type 1 diabetes: a randomized clinical trial. JAMA 2013;310:1240-1247.

34. Choudhary P, Olsen BS, Conget I, et al.: Hypoglycemia prevention and user acceptance of an insulin pump system with predictive low glucose management. Diabetes Technol Ther 2016;18:288-291.

35. Zhong A, Choudhary P, McMahon C: Effectiveness of automated insulin management features of the MiniMed ${ }^{\circledR}$ 640G sensor-augmented insulin pump. Diabetes Technol Ther 2016;18:657-663.

36. Juvenile Diabetes Research Foundation Continuous Glucose Monitoring Study Group, Beck RW, Lawrence JM, et al.: Quality-of-life measures in children and adults with type 1 diabetes: Juvenile Diabetes Research Foundation Continuous Glucose Monitoring randomized trial. Diabetes Care 2010;33:2175-2177.

37. Pickup JC, Freeman SC, Sutton AJ: Glycaemic control in type 1 diabetes during real time continuous glucose monitoring compared with self monitoring of blood glucose: meta-analysis of randomised controlled trials using individual patient data. BMJ 2011;343:d3805.

38. Little SA, Leelarathna L, Walkinshaw E, et al.: Recovery of hypoglycemia awareness in long-standing type 1 diabetes: a multicenter $2 \times 2$ factorial randomized controlled trial comparing insulin pump with multiple daily injections and continuous with conventional glucose self-monitoring (HypoCOMPaSS). Diabetes Care 2014;37:2114-2122.

39. Wong JC, Foster NC, Maahs DM, et al.: real-time continuous glucose monitoring among participants in the T1D exchange clinic registry. Diabetes Care 2014;37: 2702-2709.

40. Bailey T, Bode BW, Christiansen MP, et al.: The performance and usability of a factory-calibrated flash glucose monitoring system. Diabetes Technol Ther 2015;17: 787-794.
Address correspondence to: Pratik Choudhary, MBBS, MD, FRCP Weston Education Centre Kings College London Denmark Hill London SE5 9NU United Kingdom

E-mail: pratik.choudhary@kcl.ac.uk 